\title{
LAS ESTRATEGIAS DE MARKETING Y LOS NIVELES DE PARTICIPACIÓN DE MERCADO DE LAS UNIVERSIDADES 2010*
}

\author{
MARKETING STRATEGIES AND LEVELS OF MARKET \\ PARTICIPATION OF UNIVERSITIES 2010
}

\author{
Gonzalo Oswaldo Peralta Silva ${ }^{1}$, \\ José Germán Linares Cazola ${ }^{2}$
}

\begin{abstract}
RESUMEN
El marketing se ha constituido en un instrumento infalible para que las empresas logren éxito en el mercado globalizado y altamente competitivo, en el que no hay tregua para los competidores. Este aspecto, que hasta hace algunos años, solo era de dominio de empresas privadas y las dedicadas a la producción de bienes y servicios de demanda masiva; sin embargo, en la actualidad, el marketing, cada día, gana adeptos y mayor número de empresas se ven obligadas a usarlo; de lo contrario, están condenadas al fracaso. Esto no causaría asombro, de no ser por el hecho de que entidades dedicadas a los servicios puros están aplicando planes y estrategias de marketing; instituciones públicas y privadas están sujetas a la formulación y aplicación de planes de marketing muy bien formulados y costosamente diseñados. Esto demuestra la importancia y singular credibilidad que ponen las empresas en la técnica del marketing para asegurar su presencia en el mercado.
\end{abstract}

* Recibido: 22 de marzo del 2013; aprobado: 23 de abril del 2013.

1 Licenciado en Educación, Magister en Docencia Universitaria y Doctor en Administración de la Educación. Asesor en la Asamblea Nacional de Rectores y Docente de la Universidad Nacional Tecnológica del Cono Sur. Socio fundador de la Asociación Peruana de Desarrollo de CarrerasAPDC.E-mail: gonzalo.peralta.14@gmail.com

2 Licenciado en Administración, Magíster en Administración Pública y Doctor en Gestión de la Educación. Docente de la Universidad Nacional Mayor de San Marcos (Lima), Universidad Nacional de Ancash (Huaraz), Universidad Privada San Pedro (Chimbote), Universidad Cesar Vallejo (Chimbote).E-mail: jlinares_cazola@hotmail.com 
El desarrollo de este estudio tiene como elemento a las estrategias que emplean las universidades, con el fin de compararlas con los niveles generales de participación de mercado para conocer si son o no son efectivas frente a sus metas de captación de mercado. El problema abordado trata de analizar y determinar en qué medida las estrategias de marketing influyen en la captación de mercado en las universidades y no constituyen una inversión sin retorno. El propósito del estudio consistió en determinar si las estrategias de marketing aplicadas influyen en la captación de mercado en las universidades 2010. El tipo de investigación fue correlacional; el diseño, no experimental y transeccional-causal, porque el estudio describe las relaciones entre dos variables en un momento determinado, tales como las estrategias de marketing aplicada y su nivel de participación de las universidades. Se ha determinado las estrategias de marketing de mayor eficiencia utilizadas por las universidades para que puedan captar una mayor cantidad de alumnos en su institución. Las estrategias de marketing aplicadas desde el año 2007 al 2010 fueron las de posicionamiento -cambio de imagen corporativa- de posicionamiento de marca, centrada en el cliente-marketing directo, de ajuste de precios, de desarrollo de nuevos productos y el marketing en la era digital, todas ellas expresadas en mecanismos de comunicación y promoción técnicamente diseñados y aplicados.

PALABRAS ClAVE: Marketing, estrategias, participación de mercado, planes, posicionamiento.

\section{ABSTRACT}

This cross-sectional correlational non experimental study aimed to describe the relationship between marketing strategy and student recruitment of universities. The results showed that the marketing strategies used from 2007 to 2010 were the positioning -change of corporate image-, brand positioning focus on client-direct marketing, adjustment in prices, development of new products, and the marketing in the digital age. All of them were used mostly and their benefits were of the best ones, all of them based on communication and promotion mechanisms carefully designed and applied.

KEY WORDS: Marketing, strategies, market participation, plans, positioning.

\section{INTRODUCCIÓN}

La mayoría de estudiantes egresados de secundaria buscan un centro de estudios superiores de nivel universitario entre públicos y privados. El estado solo es capaz de albergar un porcentaje mínimo de personas y muchos optan por ingresar a una universidad privada; en consecuencia, se convierte en el gran mercado objetivo de las universidades privadas, las que afrontan una competencia educativa que, hoy por hoy, es un mercado altamente competitivo e incluso muy segmentado, por lo que, las universidades se enfrentan a un mercado que deben de captar, no siendo fácil, por lo que, deben hacer uso de estrategias de marketing educativo para captar un número adecuado de alumnos y mantener su subsistencia. 
Por tal razón, en las estrategias utilizadas por las universidades privadas existe un conjunto de actividades y acciones mercadológicas que deben ser estudiadas con la finalidad de conocer el accionar y cuan fructíferas son todas estas estrategias de marketing, razón de ser del presente trabajo.

\section{MATERIAL Y MÉTODOS}

TIPO DE INVESTIGACIÓN

La investigación es correlacional porque va a asociar las variables en estudio con el propósito de conocer su relación, las estrategias de marketing y sus niveles de captación.

\section{DISEÑO DE INVESTIGACIÓN}

La investigación es no experimental y transeccional - causal porque el estudio describe las relaciones entre dos variables en un momento determinado, tales como las estrategias de marketing aplicada y sus niveles de captación de las universidades.

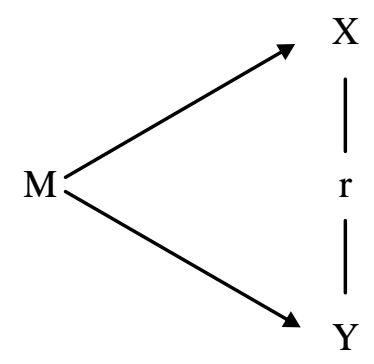

Donde:
M : $\quad$ Mercado estudiantil.
$\mathrm{X}$ : $\quad$ Estrategias de marketing.
Y : $\quad$ Niveles de participación.
$r(X \wedge Y)$ : Relación entre las variables estrategias de marketing y niveles de participación.

\section{POBLACIÓN Y MUESTRA}

La población y muestra estuvo constituida por 26043 ingresantes del 2007 al 2010 (Cuadro 01). 
Cuadro 01

INGRESANTES A LAS UNIVERSIDADES DEL 2007 AL 2010

\begin{tabular}{ccccccccc}
\hline $\begin{array}{c}\text { Captación } \\
\text { por año }\end{array}$ & \multicolumn{7}{c}{ Universidades } & \multirow{2}{*}{ Total } \\
\cline { 2 - 7 } & Univ 1 & Univ 2 & Univ 3 & Univ 4 & Univ 5 & Univ 6 & Univ 7 & \\
\hline 2007 & 770 & 1035 & 998 & 606 & 1065 & 2350 & 1015 & 7839 \\
2008 & 1680 & 1177 & 1150 & 739 & 868 & 470 & 1035 & 7119 \\
2009 & 1285 & 1950 & 1020 & 1044 & 1280 & 359 & 1260 & 8198 \\
2010 & 346 & 257 & 128 & 164 & 122 & 553 & 1317 & 2887 \\
\hline Total & $\mathbf{4 0 8 1}$ & $\mathbf{4 4 1 9}$ & $\mathbf{3 2 9 6}$ & $\mathbf{2 5 5 3}$ & $\mathbf{3 3 3 5}$ & $\mathbf{3 7 3 2}$ & $\mathbf{4 6 2 7}$ & $\mathbf{2 6 0 4 3}$ \\
\hline
\end{tabular}

Fuente: Archivo de Admisión de las universidades.

TÉCNICA E INSTRUMENTO, FUENTES E INFORMANTES

Técnicas: Luego se usó la técnica de la entrevista con un cuestionario que se aplicó a todos los alumnos ingresantes a las universidades de la capital.

Fuentes: Se ha obtenido información de:

- Fuentes secundarias: Se analizaron constancia de ingreso, ficha de matrícula, ficha socioeconómica, proporcionados por las oficinas de Admisión, Marketing, la Dirección de Tecnología de Información, Registro Académico y Caja.

- Informantes: Todos los trabajadores de las áreas de Marketing, Imagen Institucional y oficinas relacionadas con las tareas y acciones de marketing que realizan las universidades; los estudiantes egresados de institutos de preparación, colegios secundarios y postulantes a las universidades de la capital y los ingresantes de las universidades.

\section{RESULTADOS}

La captación de alumnos de las diferentes universidades de la capital, según la estrategia de Marketing aplicada en el 2010 (medido en porcentaje) indicó que la universidad 1 es la que mejores resultados ha obtenido. En cuanto a posicionamiento, la universidad 1 tuvo $28,6 \%$; la universidad 2,21,2\%; la universidad 4 , $13,8 \%$; la universidad $7,11,1 \%$; la universidad $3,10,6 \%$; la universidad 5 , $10,1 \%$; y la universidad $6,4,6 \%$. 


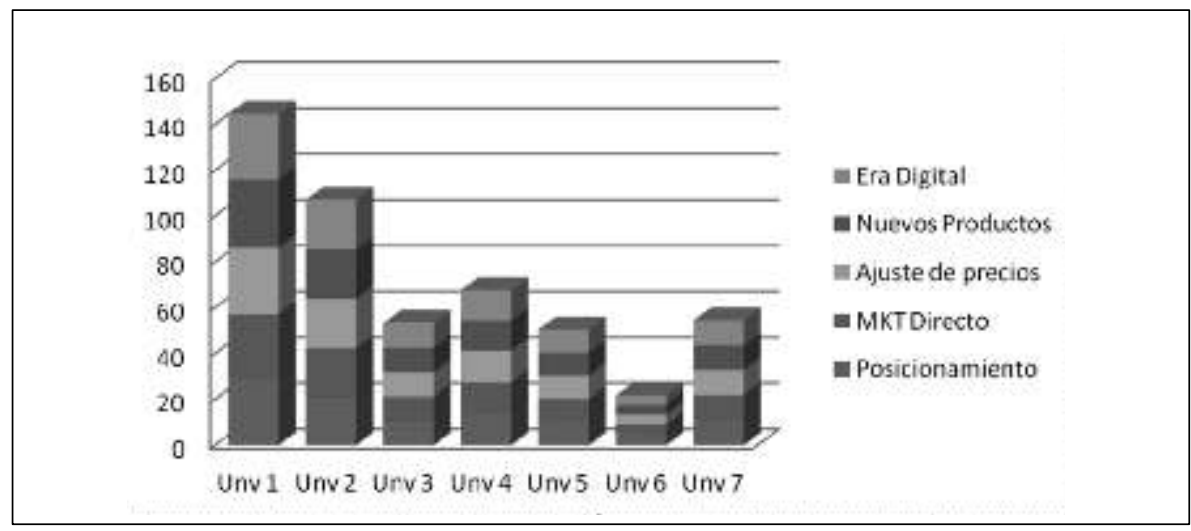

Gráfico 1. Cruce de variables: Captación de alumnos en las diferentes Universidades de la capital, según la estrategia de Marketing aplicada en el 2010, medido en porcentaje.

Fuente: Plan de marketing y archivos del área de marketing y promoción y el SPSS - v15.

\section{PRUEBA DE LA HIPÓTESIS}

Se consideraron los puntajes totales obtenidos por las variables estrategia de marketing $(\mathrm{X})$ y nivel de captación $(\mathrm{Y})$, y las siguientes hipótesis a contrastar:

\begin{tabular}{|l|l|}
\hline $\begin{array}{l}\text { Hipótesis } \\
\text { Central }\end{array}$ & $\begin{array}{l}\text { Existe una relación positiva entre la estrategia de marketing aplicada y su } \\
\text { nivel de captación de mercado en las universidades de la Capital. }\end{array}$ \\
\hline $\begin{array}{l}\text { Hipótesis } \\
\text { Nula }\end{array}$ & $\begin{array}{l}\text { No existe una relación positiva entre la estrategia de marketing aplicada y } \\
\text { su nivel de captación de mercado en las universidades de la Capital. }\end{array}$ \\
\hline
\end{tabular}

Aplicando la prueba " $\mathrm{r}$ " de Pearson mediante el programa estadístico SPSS se obtuvo el resultado siguiente:

\begin{tabular}{llcc}
\hline & & $\begin{array}{c}\text { Estrategias } \\
\text { de marketing }\end{array}$ & $\begin{array}{c}\text { Nivel de } \\
\text { captación }\end{array}$ \\
\hline Estrategia de marketing & Correlación de Pearson & 1 &. $\mathbf{9 5 4}(* *)$ \\
& Sig. (bilateral) & & .000 \\
& $\mathrm{~N}$ & 876 & 876 \\
\multirow{2}{*}{ Nivel de captación } & Correlación de Pearson & $.954\left(^{* *}\right)$ & 1 \\
& Sig. (bilateral) & .000 & \\
& $\mathrm{~N}$ & 876 & 876 \\
\hline
\end{tabular}




\section{INTERPRETACIÓN}

Con el grado de significancia $(0,954)$ se afirma que hay una correlación positiva entre las variables: estrategia de marketing (X) y el nivel de captación (Y).

\section{DISCUSIÓN DE RESULTADOS}

Es necesario tener en cuenta los resultados que nos dan a entender los cuadros, por lo tanto interpretemos cada uno de ellos.

En el cuadro 01 de la distribución de los niveles de captación por cada universidad en el año 2007 hasta el 2010, en las universidades de la capital podemos deducir lo siguiente: Que dentro de las cuatro universidades la que más creció en población o tuvo mayor captación fue la universidad 1 con un total de 4081 alumnos y la de menos captación fue la universidad 6 con un total de 3732 alumnos.

Cabe mencionar que todas las universidades no fueron de menos a más, que debería ser lo ideal; tuvieron descensos las universidades 3, 5 y 6, como se observa en el gráfico 1 . Las estrategias de marketing aplicadas desde el año 2007 al 2010 que son variadas y estructuradas según la persona al mando del área competente, se sabe que se aplicó a todas en las diferentes universidades, por igual en cada año académico, pero es evidente que sus resultados no se resaltan por igual en todas ellas. Se puede explicar entonces que las universidades que mantuvieron un nivel ascendente continuo es producto, básicamente, de la envergadura de su demanda laboral para profesionales, del bum de la construcción (para el caso de los profesionales de Arquitectura), el respaldo de la imagen corporativa y el buen performance para su proyección de su universidad; percibiéndose por esto el interés y la aceptación de los alumnos inscritos.

El caso de las universidades que sufrieron irregular avance continuo se debió al poco empuje en algunas acciones educativas que implicaban el trabajo de campo, a la falta de implementación de algunas estrategias de marketing que debieron hacerse ya en esos años, de su desnivel como actividades con relación a la era digital y el tele marketing y a la poco oferta laboral que implicaba el mercado en esos momentos. 


\section{CONCLUSIONES}

Las estrategias de marketing aplicadas desde el año 2007 al 2010, fueron de Posicionamiento, Cambio de Imagen Corporativo, de posicionamiento de Marca, Centrada en el Cliente (Marketing Directo), de Ajuste de Precios, de desarrollo de Nuevos Productos y el marketing en la era Digital.

Establecida la relación entre las estrategias de marketing y los niveles de captación de los alumnos de las diferentes escuelas profesionales de las diferentes universidades de la capital, de acuerdo a la aplicación técnica estadística dio como resultado de un grado de significancia de 0,954 .

Por lo tanto podemos decir que existe una relación positiva entre las estrategias de marketing aplicadas y los niveles de captación de alumnos de las diferentes escuelas profesionales en el periodo 2007-2010. En consecuencia, las universidades lograron mayor captación de estudiantes a través de las estrategias de marketing aplicadas.

\section{BIBLIOGRAFÍA}

\section{LIBROS:}

SIERRA, BRAVO. 1997. Técnicas de Investigación Social Teoría y Ejercicios, Decimocuarta edición, Madrid, Edit. Thomson. p. 71-9; ISBN 84283-2429-8.

HernándeZ, R.; FERnÁndez C.; BAPTISTA, P. 2006. Metodología de la Investigación; Ed. Cuarta; México D.F.; Editorial McGraw Hill. p. 277-9; ISBN 970-10-57-53-8.

Johnson, G. Y SCHOLES K. 1997. Dirección Estratégica Análisis de las estrategias de las organizaciones; tercera edición, Madrid, Editorial Prentice Hall. p. 191-9; ISBN 0-13-296849-5.

PhiliP KotLer. 1986. Mercadotecnia, tercera edición; México D.F.; editorial Prentice Hall. p. 52645; ISBN 968-880-156-9.

MCCARThy, J. Y PERREAUlt, W. 1997. Marketing, undécima edición, Madrid, Editorial McGraw Hill. p. 500-9; ISBN 0-256-10509-X.

Arellano Cueva, R. 2000. Marketing Enfoque América Latina; primera edición, México D.F., editorial McGraw Hill. p. 37-40; ISBN 970-10-2663-2.

CATEORA Graham. 2006. Marketing Internacional, duodécima edición, México D.F. Editorial McGraw Hill. p. 312-29; ISBN 10:970-10-5752-X.

Kotler, P. y Keller K. 2006. Dirección de marketing, duodécima edición, México D.F. Editorial Prentice Hall. p. 35-40; ISBN 0-13-145757-8.

DAVID, FRED R. 1997. Conceptos de administración estratégica, quinta edición, México D.F.; Editorial Prentice Hall. p.54-62; ISBN 968-880-796-6. 


\section{PÁGINAS WEBS:}

BÄVERSTAM OSCAR Y LARSSON MARIA (2009). Un estudio comparativo de la comercialización de cómo afecta a las empresas verdes dentro de la estrategia de negocio a negocio, Suecia, Departamento de Administración de Empresas y Ciencias Sociales de la Luleå University of Technology. [accesado 23 de marzol 2010], Disponible en: http://translate.google.com.pe/ translate?hl $=$ es\&langpair $=$ en $\% 7 C e s \& u=h t t p: / /$ epubl.luth.se/1402-1773/2009/123/LTUCUPP-09123-SE.pdf

Cobo Quesada Francisco Benjamín y GonZÁlez Ruiz Lasdilau (2007). Las implicaciones estratégicas del marketing relacional: fidelización y mercados ampliados. Anuario Jurídico y Económico Escurialense, X L (2007) 543-568 / ISSN: 1133-3677. [accesado 23 de marzo 2010], Archivo PDF.

GonzÁlez De Durana Covadonga Aldamiz-Echevarria (2000). La gestión de marketing en ONG's: Analisis de sus particulares y propuestas de actuación. País Vasco, Facultad de CC. Económicas y Empresariales Avda. Del Lehendakari Aguirre, 8348015 Bilbao, [accesado 23 de marzo 2010], Disponible en: http://solidaridad.universia.es/aula/formaci\%C3\%B3n/ tesis_cuatro.htm

Kitaw Daniel (2004). Comercialización Decision Support System (MDSS) Estudios de caso sobre ASPSC Mobil Oil \& East Africa Limited, Etiopía, Escuela de postgrado de la Universidad de Addis Abeba. [accesado 23 de marzol 2010], Disponible en: http://translate.google.com.pe/ translate $? \mathrm{hl}=$ es\&langpair $=$ en $\% 7 C e s \& u=h t t p: / /$ etd.aau.edu.et $/$ dspace $/$ handle $/ 123456789 / 1568$

SALAZAR AlCARRAZ JeSÚs MoISÉS (2009) en su tesis Desarrollo de una estrategia de marketing social orientada al incremento de competencias empresariales de las MYPE de la Región Cerro de Pasco, [accesado 24 de marzo 2010], Disponible en: http://www.cybertesis.edu.pe/sisbib/2008/salazar _aj/html/index-frames.html 